\title{
A Reliable Multimedia Collaboration System based on Home Network Environment
}

\author{
Eungnam $\mathrm{Ko}^{1}$ and Soongohn $\mathrm{Kim}^{2 *}$ \\ ${ }^{1}$ Division of Information \& Communication, Baekseok University, 115 \\ Anseo-dong, Dongnam-gu, Cheonan, Chungnam, 330-704, Korea \\ ${ }^{2}$ Department of Computer Science, Joongbu University, 101 Daehakro, Chubu-Meon, \\ GumsanGun, Chungnam, 312-702, Korea \\ ssken@daum.net,sgkim@joongbu.ac. \\ * Corresponding Author \\ A reliable requirement model for resource of muttimedia collaboration called \\ "RMCS_HNE" was proposed for situation-aware middleware such as home network \\ environment. RMCS_HNE model was used to detect and recover the resource errors for \\ smart multimedia collaboration system among aetions in distributed home network \\ environment. An example of situationgware applications is a smart home network system. A \\ smart home network system for distributed multimedia holds the promise of greatly improving \\ all forms of collecting remote data to show the model for smart home network system aims \\ at guaranteeing it through application favit tolerance.
}

Keywords: reliable cequirement model, RMCS_HNE, smart multimedia collaboration system

\section{Introduction}

After the early $1990^{\circ} \mathrm{s}$, the major basis of videoconferencing system moved to packet-based systems which operate on computer network [1,2]. However, since this new education system must be developed in a way that combines various field of technologies, meluding group communication and distributed multimedia processing which are the basis of packet based videoconferencing systems, integrated service functions such as middle ware are required to support it [3]. Distance education systems must be able to support real-time interaction, temporal/spatial synchronization and floor control for smooth interaction [4, 5]. In a ubiquitous computing environment, computing anytime, anywhere, any devices, the concept of situation-aware middleware has played very important roles in matching user needs with available computing resources in transparent manner in dynamic environments [6]. An example of situationaware applications is a multimedia education system. Education system for distributed multimedia holds the promise of greatly improving all forms of remote education and training [7]. This paper explains the design of the RMCS_HNE (a Reliable Multimedia Collaboration System based on Home Network Environment). DooRae supports session control, floor control, media control, window control and communication control. This paper shows a design of the agents which can detect error and recover it automatically by using API (Application Program Interface) functions in MS-windows XP or 
Windows 7 running on the situation-aware computing environment. The model aims at detecting, classifying, and recovering errors automatically. Section 2 describes situation-aware middleware as the context and QoS. Section 3 denotes the RMCS_HNE architecture and algorithm. Section 4 describes conclusion.

\section{Related Works}

As shown in Figure 1, a conceptual architecture of situation-aware middleware based on Reconfigurable Context-Sensitive Middleware (RCSM) is proposed in [8]. All of RCSM's components are layered inside a device. The Object Request Broker of RCSM (R-ORB) assumes the availability of reliable transport protocols; one R-ORB per device is sufficient. The number of ADaptive object Containers (ADC)s depends on the number of context-sensitive objects in the device. ADCs periodically collect the necessary "raw context data" through the R-ORB, which in turn collects the data from sensors and the operating system. Initially, each ADC registers with the R-ORB to express its needs for contexts and to publish the corresponding context-sensitive interface. RCSM is called reconfigurable because it allows addition or deletion of individual ADCs during runtime (to manage-new on existing context-sensitive application objects) without affecting other runtime operations inside RCSM. Ubiquitous applications require use of varous contexts to adaptively communicate with each other across multiple network environments such as mobile ad hoc networks, Internet, and mobile phone networks. However existing context-aware techniques often become inadequate in these applications where combinations of multiple contexts and users' actions need to be analyzed over a period of time. Situation-awareness in application software is considered as a desirable property to overcome this limitation. In addition to being context-sensitive, situation-aware applications can respond to both current and historical relationships of specific contexts and device-actions. An example of situation-aware applications is a multimedia distance education system running on home network environment The development of multimedia computers and communication techniques has made it possible for a mind to be transmitted from a teacher to a student in distance environment. Traditional QoS (ISO standards) was provided by the network layer of the communication system. An enhancement of QoS was achieved through coducing QoS transport services. For multimedia communication system, the QoS notion must be extended because many other services contribute to the end-to-end service quality. The organization of QoS-layered model for the multimedia communication system includes 4 layers. The four layers consist of a user QoS layer, an application QoS layer, a system QoS layer, and a network QoS layer. QoS for smart festival management system guarantees must be met in the application, system and network to get the acceptance of the users of multimedia communication system. There are several constraints which must be satisfied to provide guarantees during multimedia transmission. They are time, space, device, frequency, and reliability constraints [7]. However, it did not include multimedia collaboration control support in the architecture of situation-aware middleware for home network. In this paper, we propose a new multimedia collaboration control mechanism in situation-aware middleware for home network. 


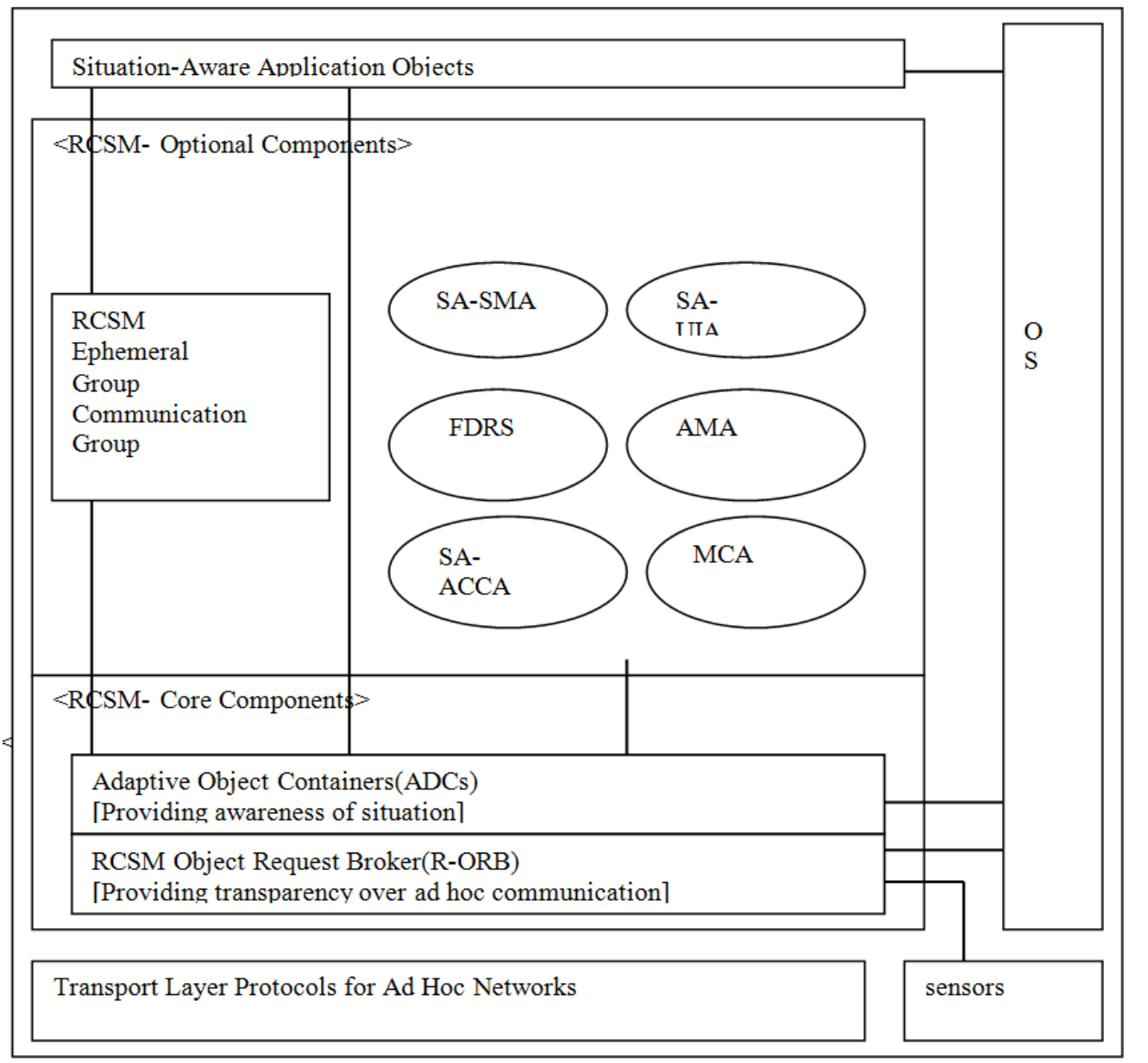

Figure 1.'RCSM's integrated components with other services

\section{The RMCS_HNE_Vodel}

\subsection{Home Network with RCSM-Optional Component and Other Services}

As shown in Figure 2, a communication layer consists of home network environment. The communication network is being presently developed with UDP broadcasting in order to decrease communication rate and TCP/IP on the Ethernet and ATM. Additional packe form has been defined and expanded for realization of multimedia collaboration's functions. The hardware environment of multimedia collaboration system consists of multimedia PCs, a network adapter, keyboard/mouse, image scanner, microphone, video camera, monitor, speaker, printer, video processor and accelerators. The operating system was first developed on Windows NT but presently Windows XP, and Windows 7 are supporting the development as well. The multimedia application layer includes general application software such as word processors, presentation tools and so on. 


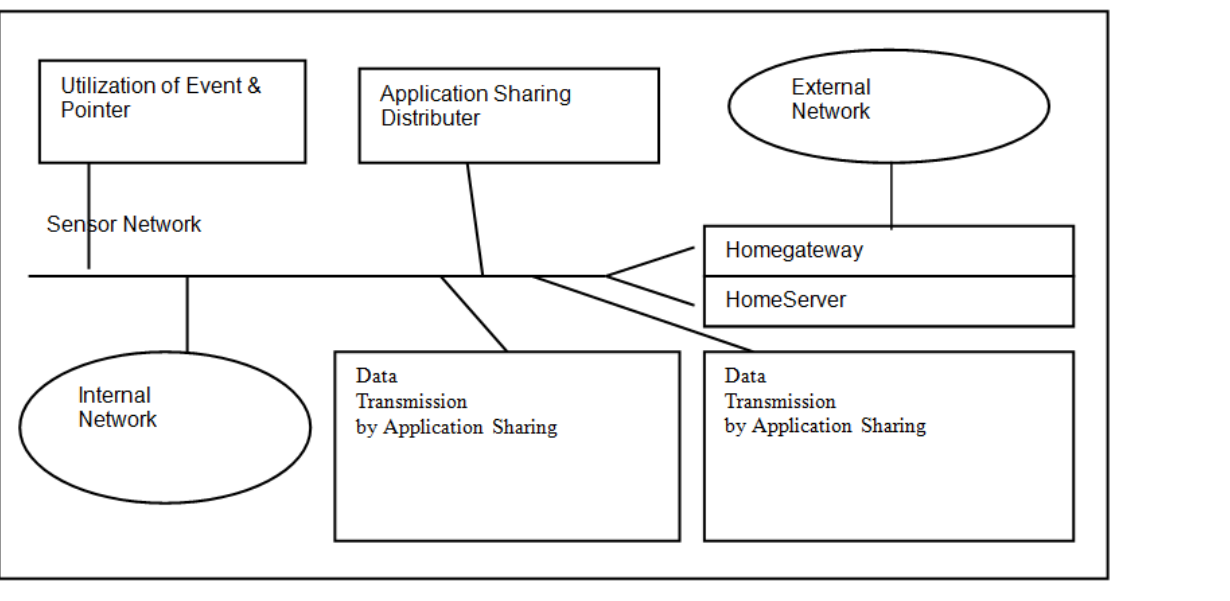

Figure 2. Home Network Environment with RCSM's integrated components

The hardware environment of multimedia collaboration system consists of multimedia PCs, a network adapter, keyboard/mouse, image scanmer, microphone, video camera, monitor, speaker, printer, video processor and accelerators. The operating system was first developed on windows NT but presently Windows XP, and Windows 7 are supporting the development as well. The multimedia application layerincludes general application software such as word processors, presentation tools and so on. As shown in Rigure 1, other services in RCSM have many agents for smart home network system. The organization of RMCS_HNE includes 4 layers. They consist of a communication layer, a system layer, a multimedia collaboration agent layer and an application layer.

\subsection{RCSM-Optional'Component and Other Services for Home Network: RMCS_HNE}

RMCS_HNE model consists of home network manager, home network adaptation, and home network for monitoring smant home network system for error detection and recovery. Figure 3 depicts the modular architecture about error data flow of RMCS_HNE model components based on smart home network system. They are implemented to permit dynamic installation and updating or existing functions even while the RMCS_HNE model for smart home network system is operating for error detection and recovery. Proxies are the principal middleware components that monitor and control the resources locally to the active nodes. They can install themselves permanently on new hosts taking part in active paths and their migration is typically single-hop. On the contrary, processors are transient and session/flowdependent gomponents that have to propagate from the client toward the server by carrying the site requirements of client user/device for that specific service flow [8]. The home network monitoring module for smart home network system has the duty of observing the state of resources and services that are local to its hosting node for error detection and recovery. The admission control module for smart home network maintains resource allocation information about all VoD flows currently served for error detection and recovery. The accounting module exploits the monitoring functions to keep a local log of the site level actually provided to the different receivers. The home network adaptation module for smart home network system is responsible for any transformation of data depending on the negotiated site level for error detection and recovery. The home network module for smart home network system coordinates the other modules and decides the site levels for the RMCS_HNE components in the VoD path for error detection and recovery. 


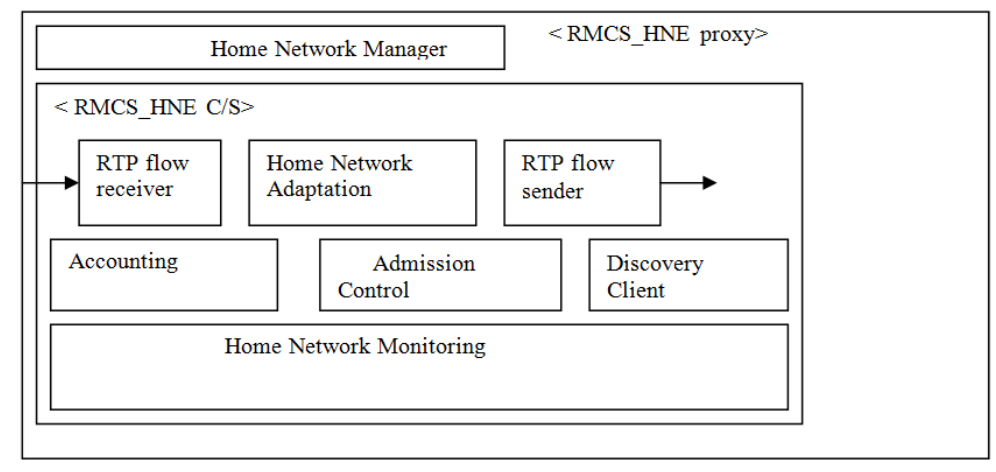

Figure 3. RMCS_HNE Model Architecture fir Smart Home Network EnVironment

\section{Simulation Results}

As shown in Table 1, conventional systems for multinedia collaboration environment are Shastra, MERMAID, MMconf, and CECED. You can seethe characteristic function of each system function for smart home management system.

Table 1. Analysis of Conventional.Mutimedia colfaboration environment for smart home network system

\begin{tabular}{|c|c|c|c|c|}
\hline Function & ShaStra & MERMAID & MiMconf & CECED \\
\hline OS & UNIX, & UNIX & UNIX & UNIX \\
\hline Development & Purdue & NEC, & CamBridge & SRI, \\
\hline Location & $\begin{array}{l}\text { Uniry. } \\
\text { USA }\end{array}$ & JAPAN & USA & International \\
\hline $\begin{array}{l}\text { Development } \\
\text { Year }\end{array}$ & 1994 & 1990 & 1990 & 1993 \\
\hline Structure & & $\begin{array}{l}\text { Server } \\
\text { /client }\end{array}$ & $\begin{array}{l}\text { Centralized } \\
\text { or } \\
\text { Replicated }\end{array}$ & $\begin{array}{l}\text { Repli- } \\
\text { cated }\end{array}$ \\
\hline protocol & ТСР/य & TCP/IP & TCP/IP & $\begin{array}{l}\mathrm{TCP} / \mathrm{IP} \\
\text { multicast }\end{array}$ \\
\hline $\begin{array}{l}\text { Error Control } \\
\text { Function } \\
\text { running on } \\
\text { RCSM for hom } \\
\text { netwo K system }\end{array}$ & $\mathrm{No}$ & No & No & No \\
\hline $\begin{array}{l}\text { Multumedia } \\
\text { Collaboration } \\
\text { Function } \\
\text { running on } \\
\text { RCSM for hom } \\
\text { network system }\end{array}$ & No & No & No & No \\
\hline
\end{tabular}

\section{Conclusions}

This paper proposes a reliable collaboration system in situation-aware middleware framework such as home network environment and presents its simulation model of agentbased. It provides several error detection and recovery functions and features capable of developing multimedia distant system among users during data flow. It is a system that is 
suitable for detecting and recovering software error based on a multimedia collaboration environment by using software techniques. There are several constraints which must be satisfied to provide guarantees during multimedia transmission. They are time, space, device, frequency, and reliability constraints. We proposed a method for increasing reliability through an adaptive reliable QoS for resource errors model for ubiquitous computing environments such as home network system. The model for smart home network system aims at guaranteeing it through application QoS. QoS for smart home network system guarantees must be met in the application, system and network to get the acceptance of the users of multimedia communication system.

Our future works are QoS-aware middleware of ubiquitous and heterogeneous environments for smart home network system.

\section{References}

[1] F. Fluckiger, "Understanding Networked Multimedia-Application and Technology" Prentice Hall Inc., Herfordshire (UK), (1995).

[2] C. W. Loftus, E. M. Sherratt, R. J. Gautier, P. A. M.Grandi D. E. PÁice and M. D. Tedd, "Distributed Software Engineering-The Practitioner Series", Prentice Hall Inc., Herfordshire (UK), (1995).

[3] ITU-T Recommendation T.122 Multipoint Communication Service for Audiographics and Audiovisual Conferencing Service Definition, ITU-T SG8 Interim Meeting $18^{\text {th }}$ oct 1994 , mertlesham, (1995) March 14.

[4] J. Y. Ahn, G. C. Park and D. J. Hwang, "Å Dynamic Window Binding Mechanism for Seamless View Sharing in Multimedia Collaboration", Proceedings of $14^{\text {th }}$ TSATED International Conference, Innsbruck Austria, (1996) February.

[5] G. C. Park, Dae J. Hwang, Design of Mmurtimedia distance learning system: MIDAS, Proceedings of the ISATED international conference, Pittsburgh USA, (1995) April.

[6] S. S. Yau and F. Karim, "Contention-Sensitive Miđdleware for Real-time Software in Ubiquitous Computing Environments", Proc. $4^{\text {th }}$ IEEE Int'l Symp. on Object-Oriented Real-time Distributed Computing (ISORC 2001), (2001) May, pp. $163-170$

[7] P. W. Agnew and A. S. Kellerman, "Distributed Multimedia", ACM Press, (1996).

[8] S. Yau, F. Karim, Y. Wang, B. Wang and S. Gupta, "Reconfigurable Context-Sensitive Middleware for Pervasive Computing" IEEE Pervasive Computing, vol. 1, no. 3, (2002) July-September, pp. 33-40.
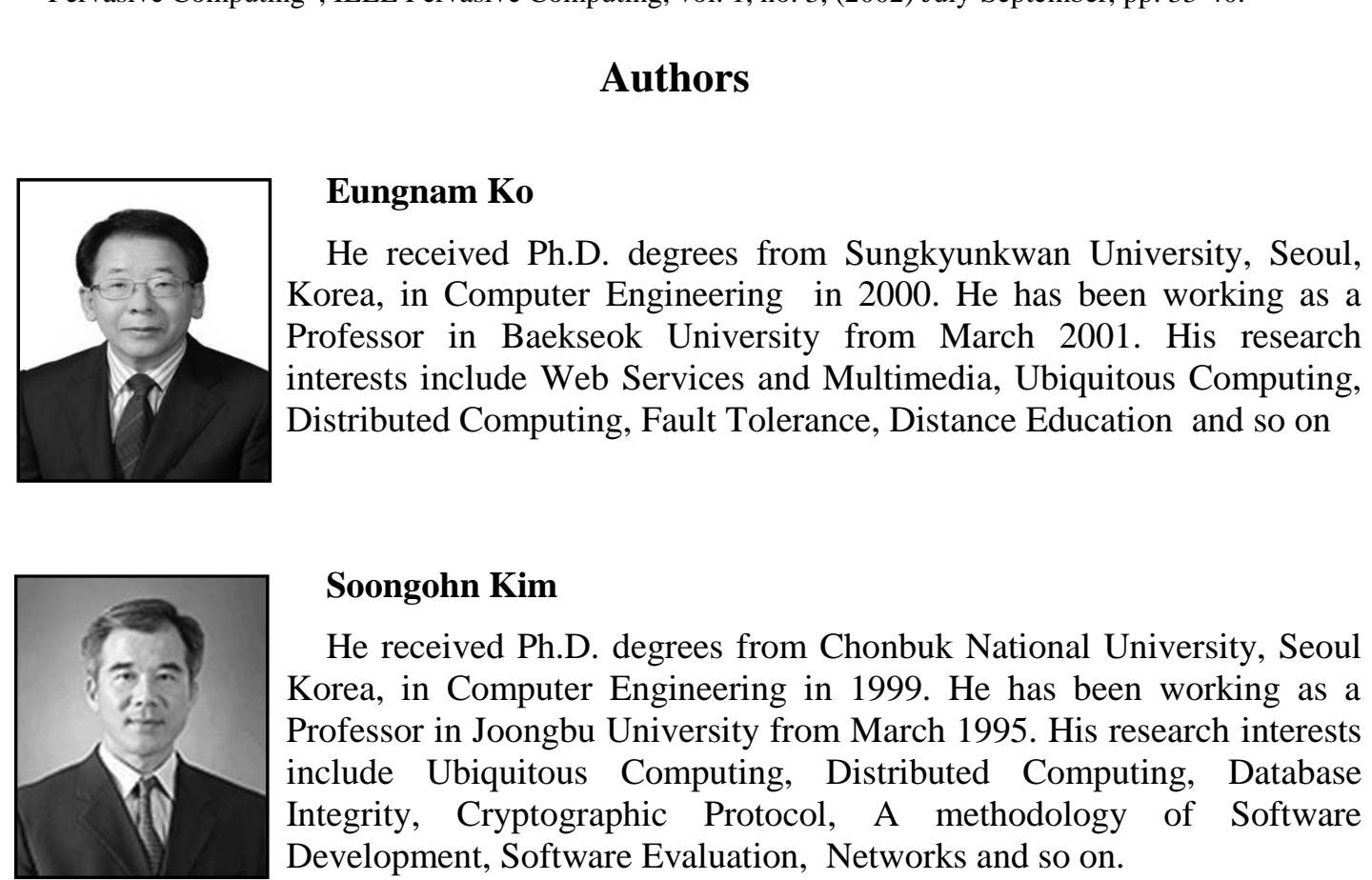

\section{Soongohn Kim}

He received Ph.D. degrees from Chonbuk National University, Seoul Korea, in Computer Engineering in 1999. He has been working as a Professor in Joongbu University from March 1995. His research interests include Ubiquitous Computing, Distributed Computing, Database Integrity, Cryptographic Protocol, A methodology of Software Development, Software Evaluation, Networks and so on. 